\title{
A falta de informação e os possíveis riscos sobre o uso exagerado da pílula do dia seguinte (levonorgestrel)
}

\author{
Lack of information and possible risks about overuse of the next day pill (levonorgestrel) \\ Falta de información y posibles riesgos sobre el uso excesivo de la píldora del día siguiente \\ (levonorgestrel)
}

Recebido: 14/09/2021 | Revisado: 20/09/2021 | Aceito: 27/09/2021 | Publicado: 29/09/2021

\author{
Ana Cristina Lima Pêgo \\ ORCID: https://orcid.org/0000-0002-9067-6164 \\ Faculdade Integrada Carajás, Brasil \\ E-mail: clima914@gmail.com \\ Sabrina da Silva Chaves \\ ORCID: https://orcid.org/0000-0001-9299-6324 \\ Faculdade Integrada Carajás, Brasil \\ E-mail: scsabrinahtmt12@gmail.com \\ Yolanda de Jesus Morais \\ ORCID: https://orcid.org/0000-0001-7105-8267 \\ Faculdade Integrada Carajás, Brasil \\ E-mail: yolandamorais123@gmail.com
}

\begin{abstract}
Resumo
Introdução: O contraceptivo de emergência foi desenvolvido inicialmente pelo médico canadense Albert Yuzpe em 1972, em que o mesmo formulou uma combinação de estrogênio e progesterona a fim de prevenir a gravidez causada por violência sexual, nesse sentido, esse artigo tem como objetivo realizar um levantamento bibliográfico detalhado sobre a falta de informação restrita e as possíveis consequências que o uso exagerado de CE pode causar á saúde da mulher, a fim de alertar principalmente os jovens e mulheres que fazem a aquisição e uso desta medicação, para que o seu uso racional e adequado possam ser promovidos evitando ou reduzindo seu uso. Método: O presente artigo foi estruturado baseando-se em uma revisão de literatura do tipo descritiva, explorando e realizando levantamentos de dados com evidências em livros e artigos científicos. Foram aplicados buscas nas seguintes bases de dados: Scielo (Scientific Eletronic Library Online), Ministério da Saúde, BVS (Biblioteca Virtual em Saúde). NIH (National Library of Medicine). Resultados e discussão: Jovens entre 15 a 20 anos de idade são as que mais consomem CE principalmente por praticarem relação sexual com ausência de preservativo, estudos apontam que os mesmos desconhecem quanto a sua farmacodinâmica e índice de eficácia máxima. Considerações finais: Os CE tem demonstrado eficácia como método de escolha de forma emergencial, desde que utilizado dentro do tempo estabelecido, ou seja, quanto mais breve for sua utilização maior será a sua eficácia. Nesse sentido, é importante destacar que a utilização desse método deve ser realizada apenas, quando for realmente necessário.
\end{abstract}

Palavras-chave: Pílula do dia seguinte; Uso irracional de levonorgestrel oral; Falta de informação adequada; Risco sobre uso frequente de contraceptivo de emergência.

\begin{abstract}
Introduction: The emergency contraceptive was initially developed by Canadian physician Albert Yuzpe in 1972, in which he formulated a combination of estrogen and progesterone in order to prevent pregnancy caused by sexual violence, in this sense, this article aims to carry out a survey detailed bibliographic information about the lack of restricted information and the possible consequences that the excessive use of EC can cause to women's health, in order to alert mainly young people and women who purchase and use this medication, so that its rational and can be promoted by preventing or reducing its use. Method: This article was structured based on a descriptive literature review, exploring and conducting data surveys with evidence in books and scientific articles. Searches were performed in the following databases: Scielo (Scientific Electronic Library Online), Ministry of Health, VHL (Virtual Health Library). NIH (National Library of Medicine). Results and discussion: Young people between 15 and 20 years of age are the ones who most consume EC mainly because they have sexual intercourse without a condom, studies show that they are unaware of its pharmacodynamics and maximum efficacy index. Final considerations: The EC has been shown to be effective as an emergency method of choice, provided that it is used within the established timeframe, that is, the sooner its use, the greater its effectiveness. In this sense, it is important to emphasize that the use of this method should only be performed when it is really necessary.
\end{abstract}


Keywords: Morning-after pill; Irrational use of oral levonorgestrel; Lack of adequate information; Risk of frequent use of emergency contraceptives.

\section{Resumen}

Introducción: El anticonceptivo de emergencia fue desarrollado inicialmente por el médico canadiense Albert Yuzpe en 1972, en el cual formuló una combinación de estrógeno y progesterona con el fin de prevenir el embarazo provocado por violencia sexual, en este sentido, este artículo tiene como objetivo realizar una encuesta bibliográfica detallada. información sobre la falta de información restringida y las posibles consecuencias que el uso excesivo de AE puede ocasionar en la salud de las mujeres, con el fin de alertar principalmente a los jóvenes y mujeres que compran y usan este medicamento, para que sea racional y pueda ser promovido mediante la prevención o reduciendo su uso. Método: Este artículo se estructuró en base a una revisión descriptiva de la literatura, explorando y realizando encuestas de datos con evidencia en libros y artículos científicos. Las búsquedas se realizaron en las siguientes bases de datos: Scielo (Biblioteca Científica Electrónica en Línea), Ministerio de Salud, BVS (Biblioteca Virtual en Salud). NIH (Biblioteca Nacional de Medicina). Resultados y discusión: Los jóvenes entre 15 y 20 años son los que más consumen AE principalmente porque mantienen relaciones sexuales sin preservativo, los estudios muestran que desconocen su farmacodinamia y su índice máximo de eficacia. Consideraciones finales: La CE ha ha demostrado ser eficaz como método de emergencia de elección, siempre que se utilice dentro del plazo establecido, es decir, cuanto antes se utilice, mayor será su eficacia. En este sentido, es importante destacar que el uso de este método solo debe realizarse cuando sea realmente necesario.

Palabras clave: Píldora del día después; Uso irracional de levonorgestrel oral; Falta de información adecuada; Riesgo del uso frecuente de anticonceptivos de emergencia.

\section{Introdução}

Os métodos contraceptivos são usados a fim de prevenir a gravidez após um ato sexual, a partir da barreira entre a penetração do espermatozoide ao óvulo, estas formas técnicas preventivas adentram-se ao planejamento familiar disponibilizado pelo Ministério da Saúde, constituídas por fatores socioculturais, antropológicos e biológicos (Remígio Souza et al., 2016). De acordo com a Pesquisa Nacional de Demografia e Saúde da Criança e da Mulher (PNDS), de 2006, existem vários tipos de contracepção disponíveis no mercado atual, os meios de contracepção e prevenção incluem métodos hormonais: Anticoncepcionais orais, barreira: Preservativos (camisinha), definitivos: Laqueadura Tubária, dispositivos intrauterinos: (DIU- Cobre) e natural: Método do muco cervical. (Brasil, 2009).

Entre os métodos contraceptivos supracitados o uso de contraceptivo de última escolha vem se tornando preocupante pela administração frequente e a ausência de consentimento de um profissional de saúde qualificado, o que acarreta no uso exagerado e incorreto desta medicação.

O contraceptivo de emergência foi desenvolvido inicialmente pelo médico canadense Albert Yuzpe em 1972, em que o mesmo formulou uma combinação de estrogênio e progesterona a fim de prevenir a gravidez causada por violência sexual. Com o passar dos anos este método foi aprovado e disponibilizado ao Brasil em 1996 pelo Ministério da Saúde e também foi incluso nas normas técnicas do programa de planejamento familiar, inicialmente por volta de 1998 foram disponibilizadas formulas constituídas de etinil estradiol e progesterona baseado no método de Albert Yuzpe, porém havia várias reclamações do medicamento e bastantes efeitos colaterais, foi assim que no ano de 1999 o contraceptivo de emergência foi reformulado contendo somente a progesterona que atualmente chama-se levonorgestrel reduzindo seus efeitos indesejáveis (Ragland et al., 2009).

Nos dias atuais mulheres em especial jovens em idade e vida sexual precoce tendem a utilizar contraceptivo emergencial sem conscientização de um profissional responsável e exacerbadamente (Barros et al. 2019).

A utilização inadequada de contraceptivo de emergência é continua, ainda que haja conhecimento deste medicamento o seu uso constante principalmente pelos jovens, podem resultar em gestações inesperadas de forma generalizada, seja por erros, interrupções de outros meios de anticoncepção ou casos inoportunos, e o escanteio de outros métodos de prevenção mais indicados e seguros para saúde do organismo feminino (Almeida et al., 2018). 
Os contraceptivos emergenciais, incluídos na classe hormonal, são medicamentos de utilidade restrita, a sua fórmula atua sob o sistema endócrino composto por hormônios concentrados em intervalo de tempo de 72 h, após o vínculo sexual. A fim de impedir a fecundação em situações como: Relações sexuais desprovidas, omitir o uso de outro anticoncepcional de rotina e abuso sexual (Brasil, 2014).

A pílula do dia seguinte não é um anticoncepcional de uso diário, e mesmo diante de todos os meios de prevenção disponíveis, as mulheres adolescentes ainda continuam a consumir em exagero o CE de ultima escolha, e nesse uso continuo deixa duvidas quanto á sua eficácia na prevenção de gravidez e por se tratar de um medicamento de alta carga hormonal é possivel que o mesmo consumido acima do recomendado possa acarretar em riscos indesejaveis e complicações para o organismo feminino. Os profissionais da saúde devem frisar mediante á uma orientação adequada no momento de dispensação e deixar no ar aos orgãos governamentais se o fácil acesso a pílula do dia seguinte é uma vantagem quando se trata da saúde da mulher.

Por tratar-se de um assunto recorrente e de pratica comum é importante ressaltar que se trata de um medicamento de emergência, não sendo indicado seu uso acima do recomendado, porém seu fácil acesso torna-se isto inaplicável deixando sujeito á saúde da mulher sobre possíveis riscos indesejáveis.

É válido destacar a importância da participação dos profissionais da saúde incluindo o farmacêutico sendo o responsável pela dispensação desta medicação, fomentar ações em conjunto com o Ministério da Saúde de conscientização e educação sexual de forma dinâmica e inovadora entre os jovens, orientando sobre o uso correto e consciente da medicação administrada enfatizando assim suas contraindicações e as possíveis reações indesejáveis.

Nesse sentido o artigo apresenta como objetivo realizar um levantamento bibliográfico detalhado sobre a falta de informação restrita e as possíveis consequências que o uso exagerado de CE pode causar á saúde da mulher, a fim de alertar principalmente os jovens e mulheres que fazem a aquisição e uso desta medicação, para que o seu uso racional e adequado possam ser promovidos evitando ou reduzindo seu uso.

\section{Contraceptivos}

A inclusão, e a disponibilidade aos recursos contraceptivos e preventivos para sexo feminino e masculino é essencial para determinar a ação reprodutiva brasileira. Com isto, os profissionais de saúde habilitados aos serviços correspondentes atuam de tal forma a auxiliar ao usuário o método contraceptivo mais ajustável à sua maneira adequada, para que se torne um caminho progressivo. Desta forma o Planejamento Familiar foi estabelecido no ano de 1996 pelo Congresso Nacional associado á Presidência da República. O Sistema Único de Saúde (SUS) tem o dever de oferecer acesso à população de vida sexual ativa, auxílio integral, prestação de serviços da saúde, métodos e informações de contracepção ou gestação, incluindo meios de esterilização disponibilizados pelo Ministério da Saúde. Logo, estes procedimentos quando realizados de forma impulsiva e incorretamente são atribuídos de acordo com as legislações vigentes consequências vigorosas. Os contraceptivos são meios que de certa forma atuam na prevenção da gravidez indesejada e regulação do ciclo menstrual (Brito; Nobre \& Vieira, 2009).

Os meios de anticoncepção são destinados em impedir o processo de ovulação, tornando o organismo feminino suscetível a passar por alterações, sendo os mais utilizados por mulheres em fase de reprodução, os anticoncepcionais de classe hormonal, incluindo o CE (Brasil, 2014).

Os contraceptivos atuam de forma eficaz quando relacionado ao seu mecanismo de ação e seu objetivo indicado, porém o consumo incorreto pode acarretar danos ao organismo feminino drasticamente como gestação precoce e inesperada, abortos negligenciados e até mesmo exposição á doenças sexualmente transmissíveis (Vieira et al., 2006). 
A aplicação dos contraceptivos pode ocorrer de várias formas, bem como: enteral, parenteral, implante introduzido sob o sistema uterino, e adesivos dérmicos (Rathke et al., 2001).

Um dos meios de anticoncepção restrito a ser desenvolvido foi hormonal à base de estrogênio e progesterona por Albert Yuzpe em 1972, com objetivo á gestação profilática de ocorrências inoportunas. Em 1998, foi aprovado para distribuição o kit de contraceptivos emergenciais (Preven), atribuído com teste de gravidez e 4 pílulas de contraceptivo azul claro inspirado no regime de Yuzpe (etinilestradiol e levonorgestrel), havia informações do paciente e instruções de uso, porém havia bastantes reações indesejáveis. Houve então, o segundo método criado chamado em plano B em que na sua fórmula continha progestógeno isolado aprovado em 1999. O ativo supracitado se tornou mais eficaz e minimiza efeitos colaterais como refluxos, cefaleia e vômitos, atualmente chamam-se de contraceptivo emergencial e o kit não são mais comercializados (Ragland; West, 2009).

\section{Contraceptivos de uso emergencial}

A busca pelo medicamento de uso restrito oral para prevenir a gravidez é de extrema preocupação, pois o uso exacerbado por mulheres tem-se tornado presente na população.

Uma das justificativas pode estar relacionada ao fácil acesso do mesmo em comércios que possuem autorização em venda isento de prescrições do profissional habilitado, o que negligência os regimes estabelecidos pela Agência Nacional de Vigilância Sanitária (Ribeiro; Silva \& Barros, 2020).

Este medicamento é chamado popularmente como: Pílula do dia seguinte, e são vendidos extremamente para prevenção de gestação indesejada, possuem garantia de eficácia comprovada, auxiliando em casos de esquecimento ou utilização incorreta do anticoncepcional de rotina, e violência sexual. Substâncias hormonais como (Levonorgestrel, ou Progestágeno) são ingeridas posteriormente á um vinculo sexual em intervalo máximo de 72 h (Souza et al., 2016).

No Brasil, o CE passou a ser disponibilizado para a população nos anos de 1999, de inicio em drogarias comunitárias sendo necessária a apresentação da prescrição e logo após, o mesmo passou a ser disponibilizado em redes de saúde pública como na Unidade Básica de Saúde (UBS) sendo medicamento isento de prescrição (Almeida et al., 2015).

O CE oral é um assunto atualmente comentado por fatores principais como o seu uso irracional e indiscriminado, adotado popularmente como pílula do dia seguinte e seu uso é de extrema cautela, pois, são indicados apenas para casos de ultima escolha no que se refere á prevenção de gravidez indesejada (Reis et al., 2017).

Os anticoncepcionais orais de uso restrito possuem grande teor hormonal, quando subjugada aos de consumo diário. Este medicamento de uso restrito é considerado como sinal vermelho por ter grandes possibilidades de prejudicar o organismo feminino por consequência do seu uso irracional e exacerbado, não se aplicando a verdadeira finalidade deste medicamento (Reis et al., 2016).

O CE oral é de extrema eficácia, mas em contrapartida o seu consumo exacerbado poderá acarretar sequelas indesejáveis ao organismo feminino, como: câncer cervical, mamário e esterilidade. É importante transmitir a informação de que este medicamento utilizado irracionalmente e repetitivamente, pode inclusive trazer consequências como uma gestação inesperada (Brasil, 2014).

O princípio ativo (Levonorgestrel) possui $1,5 \mathrm{mg}$ somente em um comprimido de dose unitária, sendo o CE mais utilizado e disponibilizado para população através da Organização Mundial da Saúde (Brasil, 2012).

Atualmente são disponibilizados mais de um tipo de CE no mercado, além do em dose única de somente 1 comprimido, contém outro presente contendo 2 comprimidos que devem ser tomados em um período de $12 \mathrm{em} 12 \mathrm{~h}$, ambos devem ser usados em tempo máximo de $72 \mathrm{~h}$ depois do ato sexual desprovido, possuindo alta eficácia de aproximadamente 98\% e segurança em uso correto e racional (Ribeiro; Silva \& Barros, 2020). 
O anticoncepcional de uso restrito somente deve ser utilizado em situações inoportunas como referência: Abuso sexual, ato sexual desprovido, ou falha de algum outro meio de prevenção de rotina (Brasil, 2014).

Novarrete et al. (2015), diante dos seus estudos esclarece de forma especifica e clara sobre o uso de CE. Descreve sua forma de concentração e posologias disponíveis, sendo pílula oral de levonorgestrel de 1,5 mg ou duas doses de pílulas da mesma de 0,75 mg, esta por sua vez a ser administrada em intervalo de $12 \mathrm{em} 12 \mathrm{~h}$, e podem ser tomadas juntas caso optar. Com base no método Yuspe são apresentadas $0,1 \mathrm{mg}$ de etinil estradiol em conjunto com 0,5 mg de levonorgestrel, seguida de uma segunda dose igual após 12 h. Há também meios disponíveis ainda na dose de Yuspe, sendo 4 pílulas compostas de anticoncepcionais orais combinados constituída de $0,03 \mathrm{mg}$ de etinil estradiol e 0,15 mg de levonorgestrel. Podem ser utilizadas também 5 pílulas de contraceptivos orais de 0,02 $\mathrm{mg}$ de etinil estradiol e 0,10 mg de levonorgestrel, ou 2 pílulas de $0,05 \mathrm{mg}$ de etinil estradiol e $0,25 \mathrm{mg}$ de levonorgestrel.

Contudo, a OMS em conjunto com empresas na área da saúde sexual e reprodução, fundou em 1995 o Consórcio Internacional de Anticoncepção de Emergência (CLAE), a fim de atribuir pontos para distribuição deste método em diversos territórios do mundo, principalmente os desenvolvidos (Souza \& Brandão, 2009).

O motivo de engradecer esse processo é minimizar os índices de natalidade em níveis precoce, inesperado ou aborto inseguro, de tal forma que, certos países têm estabelecido regimes para minimizar o acesso ao contraceptivo emergencial, como citação as normas de medicamento para o sexo feminino com antecipação do vínculo sexual, e com autoridade do profissional farmacêutico para fornecimento sem prescrição médica, isto seguindo os protocolos de exigência (Pereira \& Brandão, 2012).

As grandes consumidoras deste medicamento com finalidade profilática em gestação indesejada, são jovens com vida sexual ativa precoce onde os estudos enfatizam que alguns motivos para o seu uso são levantados como o acesso isento a outros métodos de anticoncepção, e este não haver uma confiança na sua segurança e eficácia, aplica o uso incorreto do anticoncepcional de rotina e esclarecem não obter conhecimento especifico sobre os outros meios de anticoncepcionais (Ramos et al., 2018).

\section{Contraceptivo de emergência oral: levonorgestrel}

O uso exacerbado faz com que este medicamento não seja utilizado com finalidade em casos restritos, não sendo de tal modo aconselhável como uso regular, porém esta indicação é negligenciada devido à facilidade de obtê-lo em farmácias e drogaria sem prescrição, colocando em risco a saúde feminina suscetível a efeitos indesejáveis (Cavalcante et al., 2016).

Pinheiro (2017), relatou que os profissionais médicos informam que administrar o CE (Levonorgestrel) acima do recomendado altera o ciclo menstrual, e não garante que a eficácia seja $100 \%$ confiável nesses casos.

O levonorgestrel quando utilizado de forma exacerbada podem levar a redução da sua eficácia devido o seu excesso hormonal, e quanto relacionado á sua eficácia deve por sua vez, consumir o medicamento o quanto antes, não devendo ser considerado como um anticoncepcional de rotina, mas, em casos emergenciais (Ramos et al., 2018).

No estudo de Alano et al. (2012), as modificações no período menstrual da mulher são recorrentes efeitos indesejáveis como: Cefaleia, vômitos, cólicas e outros sintomas relacionados após seu uso continuo.

O levonorgestrel contendo $75 \mathrm{~g}$ é bastante consumido no Brasil por marcas conhecidas como: Diad; Poslov; Postinor entre outras (Barros \& Cunha, 2019)

O uso de levonorgestrel de $15 \mathrm{~g}$ na amamentação não é recomendado visto que pode haver transferência de concentração de hormônio do medicamento para o leite materno o que não é saudável para o bebê. Mulheres que estão amamentando e utilizam o levonorgestrel de $15 \mathrm{~g}$, só podem amamentar após $8 \mathrm{~h}$ de ingestão do mesmo, pois é o tempo 
necessário para que ocorra o processo farmacocinético no organismo, não afetando o leite materno (Trussell; Raymond \& Cleland, 2019).

O levonorgestrel de uso oral está presente na Relação nacional de Medicamentos Essenciais (RENAME), (Brasil, 2020).

Estudos britânicos revelam que mesmo com a ingestão do CE, 1 a cada 20 mulheres foram diagnosticada com uma gravidez de risco localizada na tuba uterina (Cleland et al., 2014).

Estudos revelam que o contraceptivo de emergência possui eficácia mínima inferior, nas mulheres acima do peso normal, isso através da análise de mulheres com faixa corporal mais elevado de aproximadamente $29 \mathrm{~kg} / \mathrm{m} 2 \mathrm{com}$ o uso de levonorgestrel com índice de efeito rebote de 5,8\%. (Kapp et al., 2014)

$\mathrm{O}$ uso exacerbado faz com que este medicamento não seja utilizado com finalidade em casos restritos, não sendo de tal modo aconselhável como uso regular, porém esta indicação é negligenciada devido à facilidade de obtê-lo em farmácias e drogarias sem prescrição, colocando em risco a saúde feminina (Cavalcante et al., 2016).

\section{Mecanismo de ação pílula do dia seguinte (levonorgestrel)}

$\mathrm{O}$ mecanismo de ação do CE irá depender do ciclo menstrual da mulher. Isso levando em consideração se ouve a ovulação. Caso o processo de ovulação não tenha ocorrido ainda, ou seja, antes, do pico do hormônio luteinizante (LH), o CE agirá impedindo que essa mulher venha a ovular. O hormônio, levonorgestrel acarretará em um bloqueio da ovulação inibindo a fecundação do espermatozoide. Há também uma diminuição significativa no muco cervical. O muco cervical escorre da cérvice para a vagina, fazendo com que essa secreção ajude no transporte do espermatozoide ate o ovulo. Com a ingestão do CE ocorre a diminuição do muco cervical, tornando um ambiente mais hostil, influenciando assim diretamente na motilidade do espermatozoide e ir de encontro ao óvulo nas trompas. Ao chegar na corrente sanguínea o levornogestrel age de forma a impedir ou adiar a ovulação, no transporte do espermatozoide (Finotti, 2015).

\section{O Uso irracional de medicamentos de urgência feminina riscos e complicações}

O sistema de informatização padronizada e acesso da população neste meio contraceptivo são essenciais, incluídos em grande parte do Planejamento Familiar. O uso irracional de contraceptivo emergencial ainda é presente na população, mas, com informações precisas, as mulheres de vida sexual ativa usariam corretamente esta medicação com a prescrição de um profissional adequado e/ou minimizaria a sua utilização (Dombrowsk; Pontes \& Assis, 2013).

A pílula do dia seguinte é vendida expressamente nos finais de semana e feriados e as usuárias são em grande maioria solteiras, e geralmente não possuem conhecimento necessário desta medicação de alta concentração hormonal (Reis, 2016).

De acordo com Silva et al. (2010), jovens adolescentes possuem informações irrelevantes sobre o contraceptivo de emergência por mais popular que este seja. Os mesmos autores ainda afirmam, que os jovens estudados relatam em já terem ouvido sobre o medicamento de uso restrito para prevenção de gestação indesejada, o curioso foi que em sua grande maioria não souberam dizer para qual função objetiva é o medicamento, quando ele é realmente indicado e o seu tempo máximo de eficácia.

A gravidez indesejada e abortos são constituídos pela isenção educativa sexual, a utilização incorreta de meios contraceptivos e distúrbios na gestação de adolescentes de vida sexual precoce (Nader et al., 2008).

O Contraceptivo de emergência pode resultar em gravidez não planejada, fazendo com que os jovens utilizem meios abortivos podendo causar complicações gravíssimas (Ajay et al., 2017). 
Informações de alta precisão dos profissionais da saúde de âmbito público ou privado é extremamente importante e sua ausência gera uma extrema preocupação, pois as usuárias de anticoncepcionais relatam não adquirirem orientações sobre o método a se utilizar (Paniz; Fassa \& Silva, 2005).

O período menstrual possui 28 dias de duração, abrangendo em duas etapas como: Período de proliferação que acontece depois de 14 dias e de secreção, onde o organismo feminino produz o hormônio progesterona. Ambos ocorrem normalmente nas mulheres, isento em casos de gravidez (Golan et al., 2014).

É necessário estar atenta ao período ovulatório que costuma acontecer próximo à estes 14 dias, quando o contraceptivo de emergência é administrado fora dos períodos supracitados como o seu uso no período de proliferação bloqueia o mecanismo de ovulação e no período de secreção modifica o fluido cervical produzido pelo colo uterino tornando mais espesso causando obstáculos para o transporte de sêmen ao supositório vaginal (Brasil, 2012).

O CE causa reações indesejáveis em aproximadamente 50\% dos casos como: refluxos, dor de cabeça, nos seios, e tontura (Brasil, 2012). Porém, fica no ar a impressão de que para as mulheres não possuem muito problemas, já que são bastante consumidos após a relação sexual desprovida e em casos inoportunos (Halpern; Raymond \& Lopes, 2014). E pelo seu uso exacerbado acaba dificultando o seu objetivo em relação aos períodos menstruais e de fertilização (Brasil, 2011).

Ferreira et al. (2018), argumentam em seus estudos que estudantes já consumiram contraceptivo de emergência acima do recomendado, jovens fazem o seu uso repetidamente e de forma irracional, não pensando sobre as consequências na questão de reações adversas.

Costa et al. (2020), salientam que uma grande percentagem de mulheres usam CE sem orientação adequada e que muitos destes desconhecem a posologia adequada.

Mulheres entrevistadas relataram consumir CE doses acima da recomendação adequada, ou que já utilizaram a mesma (Bastos et al., 2009).

Bauzá et al. (2018), descreve que grande parte dos jovens estudados na universidade das Ihas Baleares (UIB), na Espanha já fez o uso de CE, um dos principais motivos foram a falha de outros meios de prevenção como: Preservativo ou anticoncepcionais de uso diário oral, ou a não utilização dos mesmo, e medo de engravidarem.

No estudo de Sheffer-Mimouni et al. (2019), demonstraram que três mulheres que faziam o consumo de levonorgestrel próximo ao período ovulatório desencadearam uma gravidez ectópica, e chamam a atenção da equipe multidisciplinar para que deem mais prioridades em casos de sintomas recorrentes de cólica abdominal após o uso de levonorgestrel.

\section{Contraindicações da pílula do dia seguinte (levonorgestrel)}

Segundo a OMS é definidos uma tabela de categorias de elegibilidade clínica para o uso de anticoncepcionais orais. A categoria varia de 1 a 4, na qual cada número contem critérios clínicos. Categoria 1, o método poderá ser usado sem restrições. Categoria 2, o método poderá ser utilizado mais com restrições, tendo em vista que os benefícios superam os riscos. Categoria 3, deixa claramente explicito que só deverá ser usado somente se não tiver nenhuma outra alternativa de tratamento dentre que os riscos são mais alto que os benefícios, sendo que se optar pelo o uso desse método, é indispensável um acompanhamento medico. E por fim categoria 4 que é o método que não deverá ser usado em hipótese alguma (Oms, 2015).

As contraindicações para a CE é unicamente se houver uma gravidez confirmada, enquadrando-se na categoria 4 de elegibilidade clínica. Havendo ressalvas para pacientes com históricos de acidente vascular cerebral, tromboembolismo, enxaqueca severa ou diabetes com complicação vascular, podendo fazer o uso do método de contracepção de emergencial, enquadrando-as na categoria 2, poderá ser usado com restrição (Brasil, 2014). 


\section{Metodologia}

O presente artigo foi estruturado baseando-se em uma revisão de literatura do tipo descritiva, explorando e realizando levantamentos de dados com evidências em livros e artigos científicos (Estrela, 2018).

Foram aplicados buscas nas seguintes bases de dados: Scielo (Scientific Eletronic Library Online), Ministério da Saúde, BVS (Biblioteca Virtual em Saúde). NIH (National Library of Medicine).

Os critérios para inclusão foram artigos publicados na língua portuguesa e estrangeira, nos últimos 20 anos, ou seja, entre 2001 a 2021, utilizando como palavras-chaves: Pílula do dia seguinte; Uso irracional de levonorgestrel oral; Falta de informação adequada sobre a pílula do dia seguinte; Risco sobre uso frequente de contraceptivo de emergência.

Os artigos publicados fora do recorte temporal, e textos que não se aplicam ao objetivo de busca do tema explícito, foram excluídos.

\section{Resultados e discussão}

No estudo realizado por Oliveira \& Burci (2019), foram entrevistados profissionais de enfermagem onde os mesmos relatam que as jovens adolescentes por volta dos 15 a 20 anos de idade são as que mais consomem CE principalmente por relação sexual com ausência de preservativo e que desconhecem quanto a sua farmacodinâmica e índice de eficácia máxima.

Ainda por meio dos estudos dos autores supracitados diante das entrevistas os profissionais enfermeiros destacam que é preocupante o uso continuo do CE pelos jovens e por seu fácil acesso e a falta de importância á outros meios de prevenção mais seguros e indicados que evitam não só a gravidez, mas, também de Doenças Sexualmente Transmissíveis (DSTs).

Quanto á eficacia de CE, Fernandes, (2012), enfatiza que ocorre a redução com passar das horas, porém quando tomada dentro de $24 \mathrm{~h}$ a mesma possui eficácia de $95 \%$, e em casos de vômito após a ingestão do medicamento é necessário realizar uma nova administração.

Brandão (2017), em sua pesquisa identificou por meio de uma entrevista com os profissionais farmacêuticos, que o CE não é muito utilizado para fins emergenciais é sim como método regular, alertando dessa forma a importância do farmacêutico no cuidado, orientação e dispensação desta medicação.

Moraes et al., (2020) enfatizam que 56,1\% dos estudantes entrevistados por eles relataram o uso de CE e 11,4\% usaram mais de uma vez no ano, destacando que os possíveis efeitos indesejáveis são: distúrbios humorais, baixo libido, náuseas, vômitos, dor de cabeça, nos seios, sangramento fora do período menstrual e aumento de peso (Olsen et al., 2018).

Abreu \& Nunes (2021), entrevistaram 232 estudantes dos cursos de medicina e farmácia em que 126 destes relataram comprar o CE em drogarias e afirmam não receber orientação sobre o seu uso. A Agência Nacional de Vigilância Sanitária (ANVISA) exige a venda de CE com a apresentação de uma prescrição médica, porém esse ato não é praticado abrindo portas para automedicação de CE de alta carga hormonal (Arrais et al., 2016).

Oliveira et al., (2015), ainda enfatiza que o consumo de CE pode causar falta de ar, distúrbios do ciclo menstrual, aumento da pressão arterial, e edemas e que seu uso continuo pode reduzir a eficácia ocasionando em uma gravidez não planejada.

Leal, Rodrigues \& Darcin (2019), destacam que a gestação em jovens adolescentes requer uma atenção especial, uma vez, que a mesma na maioria das vezes é de risco, podendo resultar em abortos e mortalidade infantil materno.

O CE ainda não é considerado como abortivo, porém o seu uso deve ser realizado com cautela, pois há indícios de ter a capacidade de causar distúrbios congênitos, e para as gestantes que estão no processo de amamentação deve-se evitar o uso visto que além de bloquear a produção de leite, ocorre a eliminação do medicamento no leite materno (Oliveira \& Oliveira, 2015). 


\section{Considerações Finais}

Os CE tem demonstrado eficácia como método de escolha de forma emergencial, desde que utilizado dentro do tempo estabelecido, ou seja, quanto mais breve for sua utilização maior será a sua eficácia. Nesse sentido, é importante destacar que a utilização desse método deve ser realizada apenas, quando for realmente necessário.

A inserção do CE nos programas de saúde da mulher é extremamente importante a fim de se impedir gravidezes indesejadas e abortos inseguros. Porém, é válido destacar que o CE não previne as DSTs, e não deve ser utilizado diariamente como contracepção oral regular. O uso racional deve ser incentivado a fim de minimizar os riscos ocasionados pelo seu uso indiscriminado.

\section{Implicações práticas}

Recomenda-se a necessidade de estudos complementares quanto aos riscos potenciais do uso indiscriminado dos CE.

\section{Contribuições dos autores}

ACLP e SSC concebeu a estratégia de pesquisa em conjunto com YJM. ACLP e SSC fez as buscas bibliográficas nas bases de dados, a seleção dos artigos e a extração de dados sob a supervisão de YJM. Os autores leram e aprovaram a versão final do manuscrito. O conteúdo da revisão é de exclusiva responsabilidade individuais dos autores.

\section{Declaração de conflito de interesses}

Os autores declaram que a pesquisa foi conduzida na ausência de quaisquer relações comerciais ou financeiras que possam ser interpretados como um potencial conflito de interesse.

\section{Referencias}

Abreu, T. M. R., \& Nunes, A. T. (2021). Conhecimento sobre metodo contraceptivo de emergencia e seus efeitos indesejaveis pelas universitárias da área da saúde de uma instituição de ensino superior de Campos dos Goytacazes-RJ. Revista Científica da Faculdade de Medicina de Campos, 16(1), 7. http://www.fmc.br/ojs/index.php/RCFMC/article/view/228/240.

Ajayi, AI, Nwokocha, EE, Adeniyi, OV, Ter Goon, D., \& Akpan, W. (2017). Riscos de gravidez não planejada e uso de contracepção de emergência: uma pesquisa de duas universidades nigerianas. BMC health services research , 17 (1), 1-8. https://doi.org/10.1186/s12913-017-2328-7.

Alano, G. M., Costa, L. N., Miranda, L. R., \& Galato, D. (2012). Conhecimento, consumo e acesso à contracepção de emergência entre mulheres universitárias no sul do Estado de Santa Catarina. Ciência \& Saúde Coletiva, 17, 2397-2404. https://doi.org/10.1590/S1413-81232012000900020.

De Almeida, F. B., de Sousa, N. M. M., Barros, G. L., de Almeida, F. B., Farias, P. A. M., \& Cabral, S. A. A. O. (2015). Avaliação do Uso de Anticoncepcionais de Emergência entre Estudantes Universitários. Revista Brasileira de Educação e Saúde, 5(3), 49-55. https://www.gvaa.com.br/revista/index.php/REBES/article/view/3720.

Arrais, PSD, Fernandes, MEP, Pizzol, TDSD, Ramos, LR, Mengue, SS, Luiza, VL, ... \& Bertoldi, AD (2016). Prevalência da automedicação no Brasil e fatores associados. Revista de Saúde Pública , 50. https://www.scielo.br/j/rsp/a/PNCVwkVMbZYwHvKN9b4ZxRh/?lang=pt\&format=pdf.

Bastos, Silvia, \& de Alcântara Bonfim, José Ruben, \& Kalckmann, Suzana, \& Figueiredo, Regina, \& Lemos Fernandes, Maria Eugênea (2009). Prevenção de doenças sexualmente transmissíveis e procura da contracepção de emergência em farmácias e drogarias do município de São Paulo. Saúde e Sociedade, 18 (4), 787-799. https://doi.org/10.1590/S0104-12902009000400021..

Bauzà, ML, Esteva, M., Molina, J., Pereiró, I., Ingla, M., \& March, S. (2018). Contracepção de emergência e hábitos de risco em uma população universitária. The European Journal of Contraception \& Reproductive Health Care , 23 (6), 427-433. https://doi.org/10.1080/13625187.2018.1533547.

Brandão, E. R., Cabral, C. D. S., Ventura, M., Paiva, S. P., Bastos, L. L., Oliveira, N. V. B. V. D., \& Szabo, I. (2016). " Bomba hormonal": os riscos da contracepção de emergência na perspectiva dos balconistas de farmácias no Rio de Janeiro, Brasil. Cadernos de Saúde Pública, 32 , e00136615. https://doi.org/10.1590/0102-311X00136615.

Brasil. (2014). Ministério da Saúde. Secretaria de Atenção à Saúde. Departamento de Ações Programáticas Estratégicas. Área Técnica de Saúde da Mulher. Anticoncepção de Emergência: perguntas e respostas para profissionais de saúde/Ministério da Saúde. http://www.as.saude.ms.gov.br/wpcontent/uploads/2016/05/Anticoncep\%C3\%A7\%C3\%A3o-de-emerg\%C3\%AAncia-1.pdf.

Brasil. (2009). Ministério da Saúde. Pesquisa Nacional de Demografia e Saúde da Criança e da Mulher - PNDS 2006: Dimensões do processo reprodutivo e da saúde da criança/ Ministério da Saúde, Centro Brasileiro de Análise e Planejamento. http://bvsms.saude.gov.br/bvs/publicacoes/pnds_crianca_mulher.pdf. 
Brasil. (2002). Ministério da Saúde. Secretaria de Políticas de Saúde. Área Técnica de Saúde da Mulher. Assistência em Planejamento Familiar: Manual Técnico/Secretaria de Políticas de Saúde. $4^{\circ}$ ed. http://bvsms.saude.gov.br/bvs/publicacoes/0102assistencia1.pdf. 16, jan. 2021

Brasil. (2ф12). Ministério da Saúde. Secretaria de Atenção e Saúde. Departamento de Ações Programáticas Estratégicas. Protocolo para Utilização do Levonorgestrel. http://bvsms.saude.gov.br/bvs/publicacoes/protocolo_para_utilizacao_levonorgestrel.pdf. Acessado em 21, jan. 2021.

Brandão, E. R. (2017). O atendimento farmacêutico às consumidoras da contracepção de emergência. Saúde $e$ Sociedade, 26 , $1122-1135$. https://www.scielo.br/j/sausoc/a/VQJz7hFZ3CpChmGrHV4rqdL/abstract/?lang=pt.

Brandão, E. R., Cabral, C. D. S., Ventura, M., Paiva, S. P., Bastos, L. L., Oliveira, N. V., \& Szabo, I. (2017). Os perigos subsumidos na contracepção de emergência: moralidades e saberes em jogo. Horizontes Antropológicos, 23, 131-161. https://www.scielo.br/pdf/ha/v23n47/0104-7183-ha-23-47-0131.pdf.

Brasil. (2012). Ministério da Saúde. Secretaria de Atenção à Saúde. Departasmento de Ações Programáticas Estratégicas. Prevenção e Tratamento dos Agravos Resultantes da Violência Sexual Contra Mulheres e Adolescentes. (3 ${ }^{\circ}$ ed.) https://bvsms.saude.gov.br/bvs/publicacoes/prevencao_ agravo_violencia_sexual_mu lheres_3ed.pdf .

Brasil. (2010). Ministério da Saúde. Secretaria de Atenção em Saúde. Departamento de Ações Programáticas Estratégicas. Diretrizes nacionais para a atenção integral à saúde de adolescentes e jovens na promoção, proteção e recuperação da saúde. http://bvsms.saude.gov.br/bvs/publicacoes/ anticoncepcao_emergencia_perguntas_r espostas_2ed.pdf.

Brasil. (2020). Ministério da Saúde. Secretaria de Ciência, Tecnologia, Inovação e Insumos Estratégicos em Saúde. Departamento de Assistência Farmacêutica e Insumos Estratégicos. Relação Nacional de Medicamentos Essenciais. https://bvsms.saude.gov.br/bvs/publicacoes/relacao_medicamentos_rename_2020.pdf.

Brito, M. B., Nobre, F., \& Vieira, C. S. (2011). Contracepción hormonal y sistema cardiovascular. Arquivos brasileiros de Cardiologia, 96, e81-e89. https://www.scielo.br/pdf/abc/v96n4/aop01211.pdf..

Cavalcante, M. D. S., Soares, M. A., Feijó, C. M., \& Fonteles, M. M. D. F. (2016). Perfil de utilização de anticoncepcional de emergência em serviços de atendimento farmacêutico de uma rede de farmácias comunitárias. Revista Eletrônica de Farmácia, 13(3), 131-139. https://revistas.ufg.br/REF/article/view/37756. Acessado em: 03, abr. 2021.

Cleland, K., Raymond, E G, Westley, E., \& Trussell, J. (2014). Revisão da contracepção de emergência: recomendações baseadas em evidências para médicos. Obstetrícia clínica e ginecologia , 57 (4), 741. https://ec.princeton.edu/questions/ec-review.pdf.

Farias da Costa, R. J., Duarte, F. M., Reis da Silva, C. de N., Santos, B. de O., Coelho de Oliveira, R. A., \& Pereira de Sena, L. W. (2020). O uso de contraceptivos de emergência em estudantes de uma instituição de ensino superior de Belém, Pará. Revista Brasileira De Educação E Saúde, 10(4), 124-130. https://doi.org/10.18378/rebes.v10i4.7934.

Dombrowski, J. G., Pontes, J. A., \& Assis, W. A. L. D. M. (2013). Atuação do enfermeiro na prescrição de contraceptivos hormonais na rede de atenção primária em saúde. Revista Brasileira de Enfermagem, 66, 827-832. https://www.scielo.br/scielo.php?script=sci_arttext\&pid=S0034-71672013000600003.

Brasil. (2015). Instituto de Saúde da Secretaria de Estado da Saúde. Governo do Estado de São Paulo, membros da Rede Brasileira de Promoção de Informações e Disponibilização da Contracepção de Emergência (REDECE). Rede Brasileira de Promoção de Informações e Disponibilização da Contracepção de Emergência. pílulas anticoncepcionais de emergência, Orientações médicas e de prestação de serviços. http://www.saude.sp.gov.br/resources/instituto-de-saude/homepage/outras-publicacoes/brazil-guidelines_2015.pdf.

Fernandes, F. M. (2012). Intervenção Farmacêutica na Contraceção Hormonal e de Emergência (Master's thesis). https://recil.grupolusofona.pt/bitstream/10437/3277/1/Interven\%C3\%A7\%C3\%A3o_Farmac\%C3\%AAutica_na_Contrace\%C3\%A7\%C3\%A3o_Hormonal_e\%20_de_Emer g\%C3\%AAncia.pdf.

Ferreira, J. B., da Costa, A. P. V., \& Chagas, A. C. F. (2018). A prática do uso da anticoncepção de emergência em jovens universitárias de uma instituição privada de Campo Grande-MS. Revista Recien-Revista Científica de Enfermagem, 8(22), 3-13. https://www.recien.com.br/index.php/Recien/article/view/243.

Figueiredo, R., Borges, A. L. V., \& Bastos de Paula, S. H. (2017). Panorama da contracepção de emergência no Brasil. In Panorama da contracepção de emergência no Brasil (pp. 249-249). https://pesquisa.bvsalud.org/portal/resource/pt/ses-34259.

Finotti, M. (2015). Manual de anticoncepção. In Manual de anticoncepção (pp. 281-281). https://central3.to.gov.br/arquivo/494569/.

Halpern, V., Raymond, EG, \& Lopez, LM (2014). Uso repetido de contracepção hormonal pré e pós-coito para prevenção da gravidez. Cochrane Database of Systematic Reviews, (9). https://bvsms.saude.gov.br/bvs/publicacoes/diretrizes_nacionais_atencao_saude_ad olescentes_jovens_promocao_saude.pdf.

Kapp, N., Abitbol, JL, Mathé, H., Scherrer, B., Guillard, H., Gainer, E., \& Ulmann, A. (2015). Efeito do peso corporal e do IMC na eficácia da contracepção de emergência com levonorgestrel. Contracepção, 91 (2), 97-104. https://pubmed.ncbi.nlm.nih.gov/25528415/.

Lacerda, J., Portela, F., \& Marques, M. (2018). O Uso Indiscriminado da Anticoncepção de Emergência: Uma Revisão Sistemática da Literatura. ID on line. Revista de psicologia, 13(43), 379-386. doi:https://doi.org/10.14295/idonline.v13i43.1541.

Leal, A. V., \& Rodrigues, C. R. (2019). Atenção farmacêutica no uso de contraceptivos de emergência: uma breve revisão. https://www.mastereditora.com.br/periodico/20190704_103151.pdf.

World Health Organization. (2015). WHO medical eligibility criteria wheel for contraceptive use. https://apps.who.int/iris/bitstream/handle/ 10665/173585/9789248549250-por.pdf?ua=1.

de Oliveira, A. P. R., \& Burci, L. M. (2019). Percepção Bioética dos Enfermeiros na Administração e/ou Orientação do Uso do Contraceptivo de Emergência. Brazilian Journal of Forensic Sciences, Medical Law and Bioethics, 8(3), 165-177. https://apps.who.int/iris/bitstream/ handle/10665/173585/9789241549257-bos.pdf. 
Oliveira, M., \& Oliveira, V. (2016). Avaliação quantitativa da dispensação de contraceptivos de emergência na região de curitiba, pr, brasil, entre 2012 e 2014. Infarma - Ciências Farmacêuticas, 27(4), 248-252. doi:http://dx.doi.org/10.14450/2318-9312.v27.e4.a2015.pp248-252

Olsen, J. M., Lago, T. D. G., Kalckmann, S., Alves, M. C. G. P., \& Escuder, M. M. L. (2018). Práticas contraceptivas de mulheres jovens: inquérito domiciliar no município de São Paulo, Brasil. Cadernos de Saúde Pública, 34, E00019617. Https://www.scielo.br/j/csp/a/xwfk8vdjjctrypkxncbpvrn/abstract/?lang=pt\#.

Paniz, V. M. V., Fassa, A. G., \& Silva, M. C. D. (2005). Conhecimento sobre anticoncepcionais em uma população de 15 anos ou mais de uma cidade do Sul do Brasil. Cadernos de Saúde Pública, 21, 1747-1760. https://www.scielo.br/scielo.php?script=sci_arttext\&pid=S0102-311X2005000600022.

Paiva, S. P., \& Brandão, E. R. (2012). Contracepção de emergência no contexto das farmácias: revisão crítica de literatura. Physis: Revista de Saúde Coletiva, 22, 17-34. https://www.scielo.br/scielo.php?pid=S0103- 73312012000100002\&script=sci_abstract\&tlng=pt.

Rodrigues, M., \& Jardim, D. (2012). Conhecimento e uso da contracepção de emergência na adolescência: contribuições para a enfermagem. Cogitare Enfermagem, 17(4). doi:http://dx.doi.org/10.5380/ce.v17i4.30381

Ragland, D., \& West, D. (2009). Conhecimentos, atitudes e comportamentos de estudantes de farmácia em relação à anticoncepção de emergência. American Journal of Pharmaceutical Education , 73 (2). https://www.ncbi.nlm.nih.gov/pmc/articles/PMC2690901/.

Ramos, LDAS, dos Santos Pereira, E., Lopes, KFAL, de Araujo, ACA, \& Lopes, NC (2018). Uso de métodos anticoncepcionais por mulheres adolescentes de escola pública. Cogitare Enfermagem , 23 (3). https://revistas.ufpr.br/cogitare/article/view/55230/pdf_1.

Rathke, A. F., Poester, D., Lorenzatto, J. F., Schmidt, V. B., \& Herter, L. D. (2001). Contracepção hormonal contendo apenas progesterona. Adolesc. Latinoam, 2(2), 90-96. http://redece.org/Contracepcaohormonal2014.pdf. 06, mar. 2021.

Brandão, E. R., Cabral, C. D. S., Ventura, M., Paiva, S. P., Bastos, L. L., Oliveira, N. V. B. V. D., \& Szabo, I. (2016). " Bomba hormonal": os riscos da contracepção de emergência na perspectiva dos balconistas de farmácias no Rio de Janeiro, Brasil. Cadernos de Saúde Pública, 32, e00136615 https://www.scielo.br/pdf/csp/v32n9/1678-4464-csp-32-09-e00136615.pdf. Acessado em: 12, maio. 2021.

Ribeiro, R. S., Silva, M. S., \& de Barros, N. B. (2020). Incidência do uso indiscriminado do levonorgestrel por alunos da EEEFM 4 de janeiro, Porto Velho/RO/Incidence of the indiscriminate use of levonorgestrel by students of EEEFM 4 de janeiro, Porto Velho/RO. Brazilian Journal of Development, 6(6), 38444-38456. https://www.brazilianjournals.com/index.php/BRJD/article/view/11807.

Schmitz, A. C., Secco, M. B., Pinheiro, T. R., \& de Almeida, A. C. C. H. (2013). Conhecimento De Adolescentes Acerca Da Contracepção De Emergência. CATUSSABA-ISSN 2237-3608, 3(1), 21-32. https://repositorio.unp.br/index.php/catussaba/article/view/366.

da Silva, B. C. S., da Silva, R. A., do Carmo Ramos, E. M. F., da Silva Paixão, E. F., \& de Souza Ronconi, F. (2020). Atuação do enfermeiro frente as orientações quanto ao uso da anticoncepçao de emergência. Revista Científica da Faculdade de Educação e Meio Ambiente, 10(Especial), 21-25. http://www.faema.edu.br/revistas/index.php/Revista-FAEMA/article/view/903.

Souza, G. G., Lima, T. N. F. A., Nóbrega, M. M., \& Barreto, C. C. M. Conhecimento e uso de anticoncepcionais hormonais: o que é certo ou errado? Temas em Saúde [Internet]. 2016 [cited 2018 Nov 01]; 16 (4): 198-211. https://temasemsaude.com/wp-content/uploads/2017/01/16414.pdf.

Souza, R. A. D., \& Brandão, E. R. (2009). Marcos normativos da anticoncepção de emergência e as dificuldades de sua institucionalização nos serviços públicos de saúde. Physis: Revista de Saúde Coletiva, 19, 1067-1086. http://www.redalyc.org/articulo.oa?id=400838225009.

da Silva Tose, B., de Sousa, E. G., Bolzon, M., de Oliveira, M. P., Dalmoneck, R., \& Rolim, E. L. G. O uso excessivo do levonorgestrel por mulheres em idade fertil moradoras do municipio de Seringueiras/Ro. https://facsaopaulo.edu.br/wp-content/uploads/sites/16/2020/06/o-uso-excessivo-do-levonorgestrelpor-mulheres-em-idade-fertil-moradoras-do-municipio-de-seringueiras-ro.pdf

Trussell, J., Raymond, EG, \& Cleland, K. (2014). Contracepção de emergência: a última chance de prevenir uma gravidez indesejada. Contemporary Readings in Law \& Social Justice , 6 (2). https://ec.princeton.edu/questions/ec-review.pdf.

Vieira, L. M., Saes, S. D. O., Dória, A. A. B., \& Goldberg, T. B. L. (2006). Reflexões sobre a anticoncepção na adolescência no Brasil. Revista Brasileira de Saúde Materno Infantil, 6, 135-140. https://www.scielo.br/pdf/rbsmi/v6n1/a16v6n1.pdf.

Zucchi, R. M., Elito Jr, J., Zucchi, F., \& Camano, L. (2004). Gravidez ectópica após uso de contracepção de emergência: relato de caso. Revista Brasileira de Ginecologia e Obstetrícia, 26(9), 741-743. http://repositorio.unifesp.br/bitstream/handle/11600/2266/S0100-72032004000900011.pdf?sequence= $1 \&$ is Allowed=y. 